\title{
Optimal sequence of systemic therapy after sorafenib failure in patients with hepatocellular carcinoma
}

\author{
Sojung Han and Do Young Kim \\ Department of Internal Medicine, Yonsei University College of Medicine, Seoul, Korea
}

Keywords: Carcinoma, Hepatocellular; Sorafenib; Protein kinase inhibitors

See Article on Page 328

Hepatocellular carcinoma (HCC) is one of the solid cancers with a dismal prognosis, particularly when it is diagnosed in the unresectable/advanced stage. ${ }^{1}$ Owing to the increasing incidence of HCC in developed countries over the last decade, ${ }^{2}$ there has been a remarkable progress in the development of novel drugs for systemic treatment, enabling HCC to escape from being classified as an "orphan tumor." Indeed, several tyrosine kinase inhibitors, including sorafenib, lenvatinib, regorafenib, and cabozantinib, have been approved as first- or second-line systemic therapies for patients with unresectable HCC. ${ }^{3-6}$ Furthermore, nivolumab, which is a immune checkpoint inhibitor targeting programmed cell death protein 1 (PD-1) on cytotoxic T cells, and ramucirumab, a monoclonal antibody inhibiting vascular endothelial growth factor receptor 2 (VEGFR2), have been approved as second-line systemic therapies with an action mechanism different from that of tyrosine kinase inhibitors. ${ }^{7,8}$ With various systemic therapeutic options now available, liver specialists treating patients with HCC have to answer a new question: what is the optimal second-line systemic treatment option after sorafenib failure?

Regarding this issue, Lee et al. ${ }^{9}$ reported comparable efficacy and safety between nivolumab and regorafenib in patients with advanced disease (Barcelona Clinic Liver Center [BCLC] stage B or C) who had experienced sorafenib failure. Their retrospective study was conducted at a single institution, and most of the enrolled patients had BCLC stage C disease (96.7\%). In 102 and 48 patients who were treated with nivolumab and regorafenib, the median overall survival (OS) was 5.9 months and 6.9 months, respectively $(P=0.77)$. There was no significant difference in disease control rates between the nivolumab and regorafenib groups (50.0\% vs. 47.1\%; $P=0.58$ ), but the objective response rate with nivolumab was higher than that with regorafenib (16.7\% vs. $5.9 \% ; P=0.041)$. There was no significant difference in time to progression (TTP) between the two groups (4.0 vs. 3.3 months; $P=0.4)$. The median OS and TTP of regorafenib-treated patients in the study were lower compared to the RESORCE trial, ${ }^{5}$ possibly due to the larger proportion of patients with macrovascular invasion and BCLC stage $C$ in the current study. Some patients (5.9\%) on regorafenib had prior sorafenib intolerance, which may have lowered OS or TTP compared to the outcomes reported in the

\footnotetext{
Abbreviations:

AFP, alpha-feto protein; BCLC, Barcelona Clinic Liver Cancer; Cl, confidence interval; HCC, hepatocellular carcinoma; HR, hazard ratio; IPTW, inverse probability of treatment weighting; OS, overall survival; PD-1, programmed cell death protein 1; RESORCE, regorafenib for patients with hepatocellular carcinoma who progressed on sorafenib treatment; TTP, time to progression; VEGFR2, vascular endothelial growth factor receptor 2
}

\section{Corresponding author : Do Young Kim}

Department of Internal Medicine, Yonsei University College of Medicine, 50-1 Yonsei-ro, Seodaemun-gu, Seoul 03722, Korea

Tel: +82-2-2228-1992, Fax: +82-2-393-6884

E-mail: dyk1025@yuhs.ac

https://orcid.org/0000-0002-8327-3439 


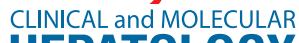

\section{HEPATOLOGY}

RESORCE trial, as longer duration of sorafenib treatment is associated with better OS and TTP with subsequent regorafenib treatment..$^{10}$ In contrast, the median OS of nivolumab-treated patients was consistent with that reported in a previous study. Independent prognostic factors for OS were nivolumab treatment (hazard ratio $[\mathrm{HR}], 0.536 ; 95 \%$ confidence interval $[\mathrm{Cl}], 0.300-0.957$; $P=0.04)$, male sex (HR, 2.587; 95\% Cl, 1.140-5.872; $P=0.02)$, Child-Pugh class B (HR, 5.195; 95\% Cl, 2.073-13.018; $P=0.001$ ), and intrahepatic tumor burden (HR, 2.801; $95 \% \mathrm{Cl}, 1.019-7.703$; $P=0.046)$. Regarding safety, patients treated with nivolumab or regorafenib had comparable toxicity leading to premature drug discontinuation, mostly from hepatic decompensation. The authors suggest that patients with Child-Pugh class B would tolerate nivolumab better than regorafenib, as there was no difference in discontinuation rates due to hepatic decompensation despite the larger number of patients with Child-Pugh class B in the nivolumab group ( $18.8 \%$ vs. $3.9 \% ; P=0.003)$.

The authors applied inverse probability of treatment weighting (IPTW) to reduce treatment selection bias, which is unavoidable in retrospective studies. In fact, the baseline characteristics were not well-balanced between the two groups. In addition to the difference in sample size ( $n=48$ in nivolumab, $n=102$ in regorafenib), a greater proportion of patients in the nivolumab group (18.8\%) had poor liver function (indicated by Child-Pugh score 7-9) compared to those in the regorafenib group (3.9\%). Additionally, the proportion of patients with intrahepatic tumor burden $>50 \%$ tended to be higher in the nivolumab group (27.1\%) than in the regorafenib group (18.6\%), although the difference was not statistically significant $(P=0.40)$. Even after IPTW, nivolumab treatment remained a significant independent factor associated with prolonged OS (HR, 0.340; 95\% Cl, 0.177-0.653; $P=0.001$ ). However, in the multivariate analysis after IPTW, nivolumab treatment was not found to be an independent factor related to prolonged TTP (HR, 0.744; 95\% Cl, 0.394-1.405; $P=0.36$ ). Based on the results obtained using IPTW, the authors concluded that nivolumab treatment might be associated with prolonged OS compared to regorafenib treatment in patients who progressed afterwards or were intolerant of sorafenib.

Although IPTW estimation is now commonly used to control for confounding factors in nonexperimental studies of medical interventions, ${ }^{11}$ not all of the confounders could be adjusted. In the study by Lee et al., ${ }^{9}$ the median duration of sorafenib treatment was 2.5 months (1.4-3.1) and 3.0 months (2.3-6.2) in the nivolumab and regorafenib groups, respectively $(P<0.001)$. Considering that longer sorafenib treatment duration is associated with better response to regorafenib, and that physicians may prefer regorafenib to nivolumab as the second-line treatment in case of a favorable response to sorafenib, there is a possibility that selection bias which cannot be corrected by IPTW may persist. Liver function and tumor burden are the strongest prognostic factors,

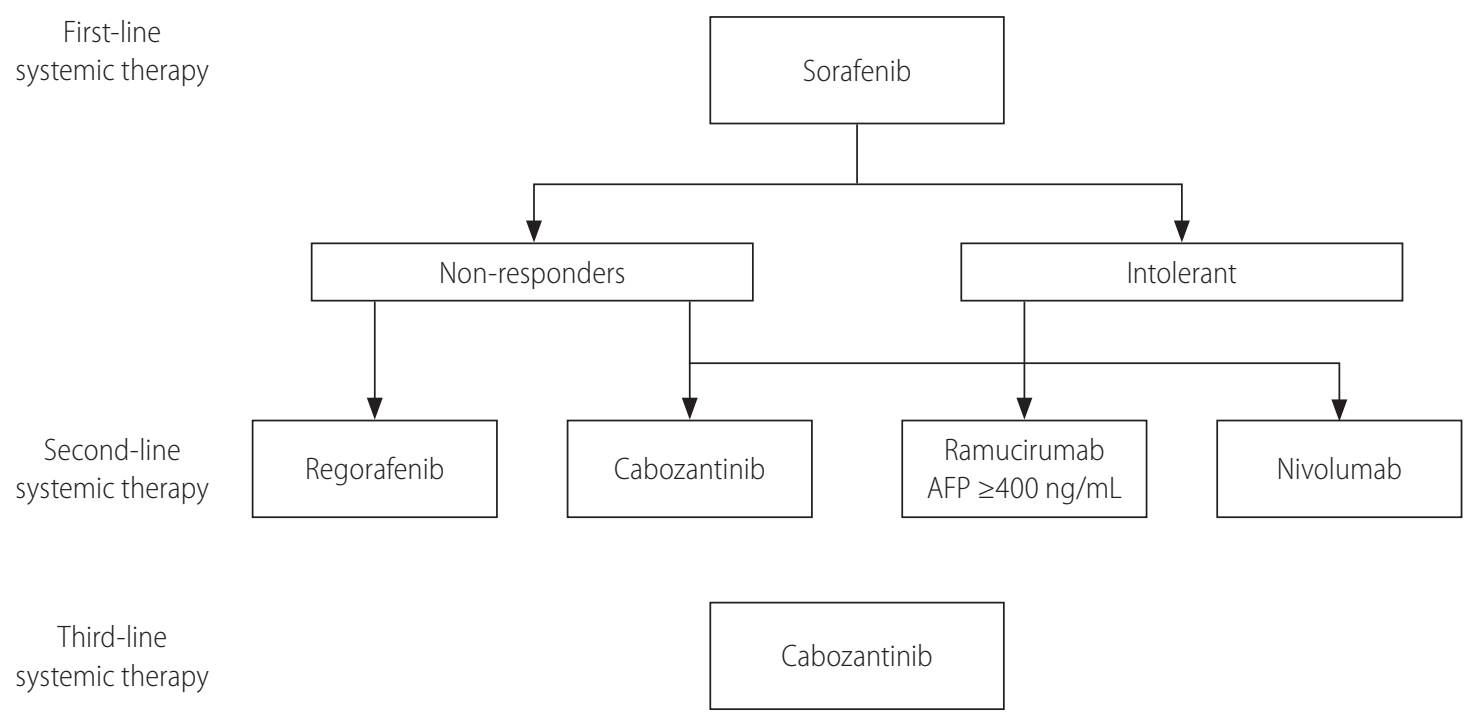

Figure 1. Proposed algorithm for the selection of systemic treatment after sorafenib failure. Potential options for sequential systemic therapies are presented. Regorafenib was the second-line therapy for patients who tolerated sorafenib ( $\geq 400 \mathrm{mg}$ of sorafenib for 20 days or longer during the 28-day period before PD) and progressed on sorafenib. Nivolumab or cabozantinib could be used for patients with intolerance to sorafenib. Ramucirumab was reserved for second-line therapy in patients with (AFP concentration $\geq 400 \mathrm{ng} / \mathrm{mL}$. Cabozantinib was the only drug listed as a third-line treatment. PD, progressive disease; AFP, alpha-fetoprotein. 
particularly in advanced HCC patients who have experienced systemic therapy. Ideally, it is desirable to conduct a randomized, controlled trial to compare the efficacy and safety between different types of systemic therapies, but it is unlikely that global pharmaceutical companies would proceed with such a trial, considering the high risk of a negative result. The alternative would be to collect nationwide large-scale data to mitigate biases such as the differences in sample size, tumor burden, liver function, and physicians' preference between groups.

With the increasing availability of drugs for first- and secondline systemic therapy for HCC, a critical question among physicians will relate to treatment strategy. What would be the optimal sequence after failure of a first-line drug, such as sorafenib? How should new drugs and data be integrated into the evolving sequence paradigm? An important hurdle to overcome is the absence of useful biomarkers to guide physicians in selecting drugs for HCC, even though recently developed drugs have molecular or immunologic targets. Unfortunately, in a recent phase 3 clinical trial testing pembrolizumab, a monoclonal antibody targeting the PD-1 interaction with programmed death-ligand 1 (PD-L1), as second-line therapy for HCC patients (KEYNOTE-240), the median OS was 13.9 months $(95 \% \mathrm{Cl}, 11.6-16.0)$ for pembrolizumab versus 10.6 months $(95 \% \mathrm{Cl}, 8.3-13.5)$ for placebo $(\mathrm{HR}, 0.781 ; 95 \% \mathrm{Cl}$, $0.611-0.998 ; P=0.238){ }^{12}$ Consequently, if similar experience is possible with nivolumab, which has been accepted as a secondline drug based on phase 1/2 data, the role of an immune checkpoint inhibitor as rescue therapy after sorafenib failure might not be so promising.

At present, a possible approach to a systemic treatment strategy can be suggested in light of the available data. Patients who were tolerant of sorafenib and had disease progression would be managed with regorafenib as second-line therapy, according to RESORCE trial. ${ }^{5}$ Cabozantinib, a multiple receptor tyrosine kinases inhibitor inhibiting VEGFR2, c-MET, and AXL, was approved as a second-line and third-line treatment for advanced HCC. In subgroup analysis, cabozantinib demonstrated favorable effects in patients aged $\geq 65$ years, males, and those with extrahepatic spread. ${ }^{6}$ Patients who discontinued sorafenib due to toxicity would be considered for nivolumab, cabozantinib, or ramucirumab. Nivolumab was tested in an open-label, non-comparative, phase 1/2 dose study (Checkmate 040) that assessed the safety and efficacy of nivolumab in patients with HCC who failed sorafenib treatment or other systemic therapy and those who were intolerant to sorafenib. ${ }^{7}$ Ramucirumab, for which survival benefit compared to placebo is not meaningful in patients who failed or were intolerant to sorafenib (8.5 vs. 7.3 months), showed improved survival in patients whose alpha-fetoprotein (AFP) concentrations are $400 \mathrm{ng} / \mathrm{mL}$ or greater. ${ }^{8}$ Therefore, ramucirumab should be restricted to patients whose AFP concentrations are $400 \mathrm{ng} / \mathrm{mL}$ or greater in both sorafenib intolerant and tolerant patients (Fig. 1). ${ }^{8}$ However, CELSTIAL trial (phase 3 double-blind placebo-controlled trial randomizing 773 HCC patients to cabozantinib or placebo in the second- or third-line setting) also reported favorable response to this particular subgroup (AFP $>400$ $\mathrm{ng} / \mathrm{mL})$; therefore, uncertainty remains on the superiority of ramucirumab over other treatment agents as second-line therapy following sorafenib failure. ${ }^{6}$ When choosing the type of systemic therapy, it is important to consider the cost-effectiveness. One study reported that cabozantinib would not be cost-effective as the second-line therapy in advanced HCC. ${ }^{13}$ In the near future, the systemic treatment paradigm will be changed as lenvatinib becomes increasingly used as a first-line therapy, and the combination of atezolizumab with bevacizumab has shown promising results as the first-line treatment in a recent phase 3 trial. With rearrangement of first-line systemic therapies in practice, the need to select the optimal second-line treatment will again be raised. It remains unclear whether individual tumor biology would help to establish predictive biomarkers in HCC and to allocate the most effective drug to the right patient. Until biomarker-driven therapy is realized, efforts should be focused on identifying the special sub-cohorts of patients who respond to individual systemic treatments.

\section{Conflicts of Interest}

The authors have no conflicts of interests to disclose.

\section{REFERENCES}

1. Craig AJ, von Felden J, Garcia-Lezana T, Sarcognato S, Villanueva A. Tumour evolution in hepatocellular carcinoma. Nat Rev Gastroenterol Hepatol 2020;17:139-152.

2. Singal $A G$, Lampertico $P$, Nahon P. Epidemiology and surveillance for hepatocellular carcinoma: new trends. J Hepatol 2020;72:250-261.

3. Llovet JM, Ricci S, Mazzaferro V, Hilgard P, Gane E, Blanc JF, et al. Sorafenib in advanced hepatocellular carcinoma. N Engl J Med 2008:359:378-390.

4. Kudo M, Finn RS, Qin S, Han KH, Ikeda K, Piscaglia F, et al. Lenvatinib versus sorafenib in first-line treatment of patients with unresectable hepatocellular carcinoma: a randomised phase 3 noninferiority trial. Lancet 2018;391:1163-1173. 
5. Bruix J, Qin S, Merle P, Granito A, Huang YH, Bodoky G, et al. Regorafenib for patients with hepatocellular carcinoma who progressed on sorafenib treatment (RESORCE): a randomised, double-blind, placebo-controlled, phase 3 trial. Lancet 2017;389:56-66.

6. Abou-Alfa GK, Meyer T, Cheng AL, El-Khoueiry AB, Rimassa L, Ryoo $B Y$, et al. Cabozantinib in patients with advanced and progressing hepatocellular carcinoma. N Engl J Med 2018;379:54-63.

7. El-Khoueiry AB, Sangro B, Yau T, Crocenzi TS, Kudo M, Hsu C, et al. Nivolumab in patients with advanced hepatocellular carcinoma (CheckMate 040): an open-label, non-comparative, phase 1/2 dose escalation and expansion trial. Lancet 2017;389:2492-2502.

8. Zhu AX, Kang YK, Yen CJ, Finn RS, Galle PR, Llovet JM, et al. Ramucirumab after sorafenib in patients with advanced hepatocellular carcinoma and increased a-fetoprotein concentrations (REACH-2): a randomised, double-blind, placebo-controlled, phase 3 trial. Lancet Oncol 2019;20:282-296.

9. Lee $\mathrm{CH}$, Lee $\mathrm{YB}$, Kim MA, Jang $H$, Oh H, Kim SW, et al. Effective- ness of nivolumab versus regorafenib in hepatocellular carcinoma patients who failed sorafenib treatment. Clin Mol Hepatol 2020;26:328-339.

10. Yoo C, Park JW, Kim YJ, Kim DY, Yu SJ, Lim TS, et al. Multicenter retrospective analysis of the safety and efficacy of regorafenib after progression on sorafenib in Korean patients with hepatocellular carcinoma. Invest New Drugs 2019;37:567-572.

11. Ellis AR, Brookhart MA. Approaches to inverse-probability-oftreatment--weighted estimation with concurrent treatments. J Clin Epidemiol 2013;66(8 Suppl):S51-S56.

12. Finn RS, Ryoo BY, Merle P, Kudo M, Bouattour M, Lim HY, et al. Pembrolizumab as second-line therapy in patients with advanced hepatocellular carcinoma in KEYNOTE-240: a randomized, doubleblind, phase III trial. J Clin Oncol 2020;38:193-202.

13. Liao W, Huang J, Hutton D, Zhu G, Wu Q, Wen F, et al. Cost-effectiveness analysis of cabozantinib as second-line therapy in advanced hepatocellular carcinoma. Liver Int 2019;39:2408-2416. 